\title{
Avaliação de Imagem Corporal em Obesos no Contexto Cirúrgico de Redução de Peso: Revisão Sistemática
}

\author{
Thiago Gomes de Castro ${ }^{1}$ \\ Marcelle Matiazo Pinhatti \\ Universidade Federal do Rio Grande do Sul, Porto Alegre, RS, Brasil \\ Rodrigo Machado Rodrigues \\ Pontifícia Universidade Católica do Rio Grande do Sul, Porto Alegre, RS, Brasil
}

\begin{abstract}
Resumo
A imagem corporal é amplamente definida como o conjunto de percepções, pensamentos e sentimentos de um indivíduo sobre o seu próprio corpo. A alteração da imagem corporal em quadros de obesidade severa é relatada consistentemente na literatura médica e psicológica. O objetivo da presente pesquisa foi investigar o instrumental de avaliação de imagem corporal utilizado em estudos com obesos no contexto de intervenção cirúrgica de redução de peso. Realizou-se uma revisão sistemática em cinco bases de dados no período de janeiro de 2009 a dezembro de 2014. Artigos em língua inglesa e portuguesa foram incluídos para análise. A busca com os descritores retornou 462 registros dos quais 15 cumpriram os critérios de inclusão. Foram identificados nove instrumentos que mensuram imagem corporal. Destacou-se a prevalência de instrumentos de autorrelato sobre a satisfação com o próprio corpo. Embora a literatura descreva a alteração perceptiva da imagem corporal em obesos, a avaliação prioriza o nível de satisfação subjetiva com o próprio corpo.
\end{abstract}

Palavras-chave: Obesidade, imagem corporal, cirurgia, revisão sistemática.

\section{Body Image Evaluation of the Obese Population in a Surgical weight Reduction Context: Systematic Review}

\begin{abstract}
Body image is broadly defined as the set of perceptions, thoughts and feelings of an individual about his own body. Changes in body image in severe obesity condition are consistently reported in the medical and psychological literature. The aim of this research was to investigate the set of instrumental assessment applied in studies of body image in obese within the context of surgical intervention for weight loss. We conducted a systematic review in five databases from January 2009 to December 2014. Articles in English and Portuguese were included for analysis. The search with the descriptors returned 462 records of which 15 met the inclusion criteria. Nine instruments that measure body image have been identified. There is a significant trend towards the use of self-report scales on perceived satisfaction of
\end{abstract}

Endereço para correspondência: Rua Ferreira Viana, no 508, Apto. 302, Bairro Petrópolis, Porto Alegre, RS, Brasil, 90670-100. Fone: (51) 3276-5257.E-mail: thiago.cast@gmail.com

Apoio Conselho Nacional de Desenvolvimento Científico e Tecnológico (CNPq) - Bolsa de Iniciação Científica Programa Institucional de Bolsas de Iniciação Científica (PIBIC; $3^{\circ}$ autor). 
body image. Although the literature describes perceptual changes in body image in obese population, its evaluation prioritizes the level of subjective satisfaction with body image.

Keywords: Obesity, body image, surgery, systematic review.

\section{Evaluación de la Imagen Corporal en Obesos en el Contexto Quirúrgico de Reducción de Peso: Revisión Sistemática}

\section{Resumen}

La imagen corporal es ampliamente definida como el conjunto de percepciones, pensamientos y sentimientos de un individuo sobre su propio cuerpo. El cambio en la imagen corporal en el marco de la obesidad severa se informa constantemente en la literatura médica y psicológica. El objetivo de la investigación fue investigar el uso de instrumentos de imagen corporal en estúdios en el contexto de la cirugía de reducción de peso en los obesos. Se realizó una revisión sistemática en cinco bases de datos a partir de enero de 2009 a diciembre de 2014. Se incluyeron artículos en Inglés y Portugués para el análisis. La búsqueda con los descriptores devuelto 462 artículos de los cuales 15 cumplieron los criterios de inclusión. Se han identificado nueve instrumentos que miden la imagen corporal. Se destaca el predominio de instrumentos de auto-informe de satisfacción con sus propios cuerpos. Aunque la literatura describe el cambio de percepción de la imagen corporal en los obesos, la evaluación prioriza el nivel de satisfacción subjetiva con la imagen corporal.

Palabras clave: Obesidad, imagen corporal, cirugía, revisión sistemática.

A obesidade é definida pela Organização Mundial da Saúde (OMS) como um distúrbio no qual o acúmulo excessivo de gordura corporal atinge uma extensão que pode afetar adversamente a saúde (World Health Organization [WHO], 1997). Estudos apontam que a etiologia da obesidade é multifatorial e que as principais causas estão relacionadas à dificuldade em perder peso devido a fatores genéticos e hábitos de vida não saudáveis (Dhurandhar \& Keith, 2014; Skelton, Irby, Grzywacz, \& Miller, 2011). Outros estudos alertam para as consequências clínicas do fenômeno, como a forte associação com diabetes mellitus tipo 2, com déficits no sistema respiratório e no sistema cardiovascular, bem como a maior incidência de câncer nessa população (Guh et al., 2009). Pesquisas indicam ainda uma relação entre obesidade e consequências psicológicas adversas (Carr \& Jaffe, 2012; Finer, 2011). A prevalência de sobrepeso e obesidade vem crescendo consideravelmente entre crianças (Skelton, Cook, Auinger, Klein, \& Barlow, 2009), adultos (Conde \& Borges, 2011) e idosos (Salihu, Bonnema, \& Alio, 2009), tanto em países desenvolvidos quanto em países em desen- volvimento (Popkin, 2007). Estima-se que em 2025 a obesidade atinja $40 \%$ da população nos EUA, $30 \%$ na Inglaterra e $20 \%$ no Brasil (Hu, 2008).

O tratamento da obesidade é condicional ao grau de severidade da concentração de gordura no corpo e às comorbidades associadas ao quadro. O método de avaliação da obesidade mais disseminado no ocidente é o cálculo do índice de massa corporal (IMC), medida utilizada pela OMS na diferenciação entre obesidade grau I (IMC $\geq 30$ e $\leq 35$ ), grau II (IMC $\geq 35$ e $\leq 40$ ) e grau III (IMC >40). No estrato de obesidade I, o manejo tradicional para a redução de peso consiste de dietas, reeducação alimentar e incentivo à rotina de atividades físicas. Contudo, evidências mais recentes têm indicado que em situações de associação da obesidade I com comorbidades, como a diabetes tipo 2, intervenções cirúrgicas são mais eficazes no manejo do peso e na saúde geral do paciente (Gianos et al., 2012). A partir do grau de obesidade II, considerado severo, o tratamento cirúrgico é consensual na literatura como padrão-ouro de intervenção, indicando maior perda de peso e manutenção dessa redu- 
ção em longo prazo (Maggard et al., 2005). Modalidades de tratamento não cirúrgicas são em geral ineficazes na redução de peso entre obesos grau II e III (Buchwald et al., 2004). A média de redução de peso em até dois anos após a intervenção cirúrgica em obesos severos é de $21.6 \%$, enquanto em tratamentos não cirúrgicos essa taxa atinge 5.5\%, com altos índices de reganho de peso (Picot et al., 2009). A cirurgia de redução de peso, em suas diferentes modalidades, é considerada a medida mais eficaz, evitando o elevado risco de vida associado a uma obesidade severa não tratada (Bruce \& Mitchell, 2014).

A obesidade pode acarretar severas consequências psicológicas (Friedman \& Brownell, 1995). Contudo, ainda que a maioria dos programas de cirurgia para obesidade reconheça a importância da avaliação psicológica no pré e pós-cirúrgico, o escopo e o propósito dessas avaliações é muito variável. Fabricatore, Crerand, Wadden, Sarwer, e Krasucki (2006) evidenciaram que a concordância entre profissionais da saúde, na área de cirurgia para obesidade, reside na contraindicação de pacientes com quadros psiquiátricos graves. De fato, indivíduos com dois ou mais diagnósticos de quadro psiquiátrico tendem a perder significativamente menos peso após a cirurgia de redução de peso (Kinzl et al., 2006).

No entanto, independentemente dos quadros psiquiátricos, processos cognitivos e psicossociais isoladamente têm sido apontados como potenciais preditores de qualidade de vida e adaptação após a cirurgia bariátrica (Pull, 2010). Assim, para além do diagnóstico clínico de transtornos psiquiátricos, a investigação de correlatos psicológicos fidedignos da obesidade deve ser incentivada. Wimmelmann, Dela e Mortensen (2014) investigaram preditores psicológicos de sucesso e fracasso cirúrgico na perda de peso e identificaram que fatores como sintomas psiquiátricos, autoestima e imagem corporal no pré-operatório foram os mais importantes para a saúde mental após a cirurgia.

Nesse conjunto de variáveis, a imagem corporal desponta como um preditor de hábitos de saúde relacionado à qualidade de vida mesmo após dois anos da cirurgia de redução de peso (van Hout, Hagendoren, Verschure, \& van Heck, 2009). Além disso, uma imagem negativa do corpo antes da cirurgia é associada com menor perda de peso no pós-operatório, sugerindo possíveis benefícios de intervenções adicionais de apoio após a cirurgia (Ortega, Fernandez-Canet, Alvarez-Valdeita, Cassinello, \& Baguena-Puigcerver, 2012). Todavia, o conceito de imagem corporal é um dos mais controversos nos protocolos de avaliação psicológica em obesos.

A imagem corporal vem sendo amplamente definida como o conjunto de percepções, pensamentos e sentimentos de um indivíduo sobre o seu próprio corpo (Cash \& Pruzinsky, 2002). Os aportes ao conceito vão desde a investigação da satisfação subjetiva com o próprio corpo (Holsen, Carlson, \& Skogbrott, 2012), passando pela representação cognitiva do próprio corpo (Altabe \& Thompson, 1996), até a estimativa perceptiva do tamanho corporal real e desejado (Liechty \& Lee, 2015). Tal variabilidade de definições acarretou na construção de diferentes instrumentos de avaliação, o que se reflete na investigação de várias facetas de imagem corporal (Thompson, 2004). Nesse sentido, os resultados de diferentes pesquisas dificilmente poderiam ser comparados, o que impediria a construção de um corpo robusto de evidências sobre a variável.

Na década de 1990, Friedman e Brownell (1995) já haviam destacado a diversificação de avaliações no que denominaram primeira geração de correlatos psicológicos da obesidade. As avaliações se dividiam até então entre depreciação da imagem corporal e distorção da imagem corporal. Mais recentemente, Pull e Aguayo (2011), revisaram a literatura de instrumentos de imagem corporal em obesos, cobrindo o período de 2007 a 2010. Os pesquisadores encontraram um grande número de instrumentos que não apresentavam índices de consistência e validade estatística. De modo geral, o instrumental na área se dividia entre: (a) avaliação de imagem corporal via uma questão sobre satisfação com o próprio peso, (b) questionários de autorrelato sobre percepção e satisfação com a imagem corporal, e (c) escalas de figura ou silhueta estáticas de imagem corporal. 
Considerando as diferentes definições e instrumentos para imagem corporal na literatura, o objetivo deste estudo foi revisar sistematicamente os artigos empíricos publicados entre 2009 e 2014 que avaliaram imagem corporal em pacientes obesos em um contexto de intervenção cirúrgica para redução de peso. Por contexto cirúrgico, optou-se por incluir aqueles trabalhos que apresentaram avaliação de imagem corporal na etapa pré-cirúrgica, pós-cirúrgica ou ambas.

\section{Método}

A revisão sistemática de literatura consiste na reunião, avaliação crítica e sintética de resultados de múltiplos estudos sobre determinado tema de pesquisa (Costa \& Zoltowski, 2014). As etapas do processo de revisão foram: (a) formulação e delimitação da questão de pesquisa, (b) escolha das fontes de dados, (c) eleição das palavras-chave para busca, (d) busca e organização dos resultados, (e) seleção dos artigos de acordo com os critérios de inclusão e exclusão, (f) extração dos dados dos artigos selecionados, (g) avaliação dos artigos, e (h) síntese e interpretação dos dados.

\section{Estratégias de Busca}

Foi realizada uma busca em cinco bases científicas eletrônicas: Scopus, PubMed, Web of Science, PsycINFO e Biblioteca Virtual em Saúde - Psicologia (BVS-Psi). Os descritores utilizados na busca foram selecionados na lista de termos psicológicos indexados pelo Thesaurus-APA (American Psychological Association), são eles: body image, morbid obesity, obesity e surgery. Uma tentativa inicial de especificação da modalidade cirúrgica de redução de peso para bariatric surgery resultou na exclusão de artigos potenciais para inclusão na análise. Os procedimentos cirúrgicos de redução de peso podem receber diferentes denominações, como adjustable gastric banding surgery, sleeve gastrectomy surgery e gastric bypass surgery. Assim, com o termo surgery, diferentes nomenclaturas para a cirurgia de redução de peso foram contempladas. Todos os termos foram pesquisados em strings de busca com o operador boleano AND confor- me a seguinte descrição: (a) body image AND morbid obesity; (b) body image AND obesity; (c) body image AND morbid obesity AND surgery, (d) body image AND obesity AND surgery. Os descritores e strings foram repetidos em língua portuguesa. Dois pesquisadores realizaram os procedimentos de inclusão e exclusão de forma independente. Nos casos de dúvida, um terceiro juiz analisou os resumos para decisão.

\section{Critérios de Inclusão e Exclusão}

Os critérios de inclusão para análise foram:

1. Ter sido publicado no período de janeiro de 2009 a dezembro de 2014,

2. Artigos publicados em língua inglesa ou portuguesa,

3. Artigos com resultados empíricos,

4. Artigos que relataram o uso específico de pelo menos um instrumento de imagem corporal, e,

5. Ter como amostra pacientes obesos adultos em contexto de cirurgia de redução de peso. Foram excluídos da seleção registros de artigos de revisão teórica, revisão sistemática de literatura, metanálises, teses e dissertações. Artigos repetidos em mais de uma base de dados foram considerados para análise no primeiro registro e nas repetições foram desconsiderados. O critério de exclusão foi aplicado inicialmente na leitura do título e resumo de cada artigo retornado na busca. Em situação de dúvida sobre a natureza do artigo, se empírico ou teórico, os juízes acessaram os textos na íntegra para reaplicação do critério de exclusão. Após este filtro, os critérios de inclusão foram aplicados a partir da análise do artigo na íntegra.

\section{Procedimentos de Análise}

Os artigos selecionados foram lidos na íntegra e analisados conforme as seguintes categorias descritivas: (a) características de peso e idade da amostra de obesos estudada, (b) características dos instrumentos de avaliação de imagem corporal, (c) momentos de avaliação da imagem corporal relativos à intervenção cirúrgica, (d) comorbidades psiquiátricas avaliadas nas 
amostras dos estudos, e (e) resultados da mensuração de imagem corporal nas pesquisas.

\section{Resultados}

A busca nas bases identificou 462 artigos. A primeira filtragem de resumos resultou na ex- clusão de 339 artigos, que em sua maioria representavam artigos repetidos entre as bases. Após esta filtragem, 123 artigos originais foram analisados na íntegra para aplicação dos critérios de inclusão, o que resultou na seleção de 15 artigos empíricos (Figura 1).

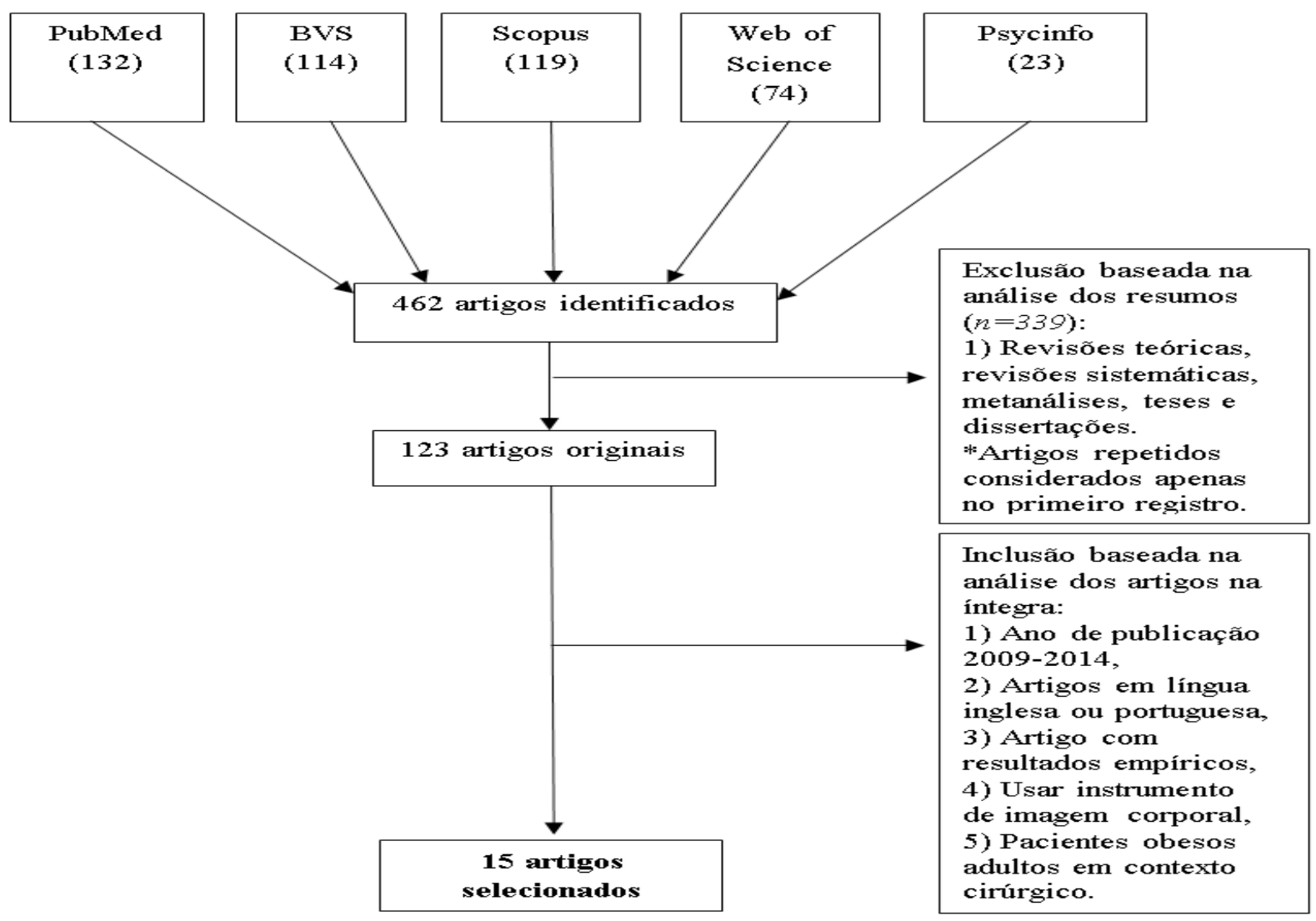

Figura 1. Fluxograma de seleção, exclusão e inclusão de artigos para análise na revisão.

A distribuição de sexo entre os participantes dos artigos selecionados foi homogênea, sendo que apenas um deles teve sua amostra composta somente por mulheres. A média de idade dos participantes dos artigos da revisão foi de 41,76 anos. Quatro estudos não especificaram a média de idade. Os estudos que mensuraram o IMC dos participantes no pré-operatório $(n=12)$ evidenciaram uma média acima de $40 \mathrm{~kg} / \mathrm{m} 2$, tipificando um padrão de obesidade grau III ou mórbida.

No conjunto de artigos selecionados, identificou-se o uso de nove instrumentos diferentes para mensurar imagem corporal. Cada estudo utilizou apenas uma medida para avaliar a imagem corporal, exceto o estudo de Koller, Schubhart e Hintringer (2013) que fez uso de duas. As escalas utilizadas em mais de $50 \%$ desses artigos foram a Body Shape Questionnaire - BSQ $(n=5)$, desenvolvida por Cooper, Taylor, Cooper e Fairburn (1987), e a Escala de Silhuetas $(n=3)$, inicialmente criada por Stunkard, Sorenson e Schulsinger (1983). Os dois instrumentos avaliam aspectos diferentes da imagem corporal. A BSQ mede a satisfação e a preocupação com o corpo e a forma corporal através de 34 itens de autorrelato organizados em uma escala Likert de seis pontos (Sousa et al., 2014). A Escala de Silhuetas, por sua vez, possui diferentes versões, 
mas a ideia central do instrumento é avaliar a satisfação e a percepção corporal dos indivíduos. Essa avaliação é realizada a partir da eleição de uma silhueta em um conjunto de figuras de si- lhueta. O participante indica aquela figura que melhor representa seu corpo atualmente e a mais próxima do ideal de seu peso (Kakeshita, Silva, Zanata, \& Almeida, 2009).

\section{Tabela 1}

Artigos Incluídos na Revisão

\begin{tabular}{|c|c|c|c|c|c|}
\hline Estudo & Amostra & Instrumentos de IC & $\begin{array}{c}\text { Momento } \\
\text { Avaliação IC }\end{array}$ & Comorbidades & Resultados IC \\
\hline $\begin{array}{l}\text { Almeida, } \\
\text { Zanatta, \& } \\
\text { Rezende, } \\
2012\end{array}$ & $\begin{array}{c}17 \text { adultos }(36,8 \pm \\
10,9 \text { anos) / IMC } \\
\text { no pré-operatório } \\
52,3 \pm 1,5 \mathrm{~kg} / \mathrm{m} 2\end{array}$ & $\begin{array}{l}\text { Escala de Figuras } \\
\text { de Silhuetas }\end{array}$ & $\begin{array}{c}\text { Pré-op / Pós-op } \\
6 \text { meses / Pós-op } \\
12 \text { meses }\end{array}$ & $\begin{array}{l}\text { Depressão } \\
\text { Leve }\end{array}$ & $\begin{array}{l}\text { Diferenças } \\
\mathrm{s} \text { i g } \mathrm{n} \text { i f i c a t i v a } \mathrm{s} \\
\text { comparando o pré-op } \\
\text { com o pós-op } 6 \text { meses, } \\
t(10)=6,59 ; p<0,01, \\
\text { e } 12 \text { meses, } t(10)= \\
10,23 ; p<0,01 / \text { Não há } \\
\text { diferenças entre pós-op } 6 \\
\text { e } 12 \text { meses. }\end{array}$ \\
\hline
\end{tabular}

Buser, Lam, \& Poplawski, 106 mulheres 2009

Grilo,

Henderson,

Bell, \&

Crosby, 2013

Kinzl,

Lanthaler,

Stuerz, \&

Algner, 2011

176 adultos

IMC médio no

(42,11 anos) /

IMC médio no

pós-op 44,46

(idade $=43,52)$ /

IMC médio no anos) / IMC médio no préoperatório 50,2 $\mathrm{kg} / \mathrm{m} 2$
180 adultos (49 anos) / IMC no pré-operatório 45 $\mathrm{kg} / \mathrm{m} 2$
Body Image State Scale

Pós-op 6-18 meses / Pós-op 19-40 meses

Depressão

(41,65 anos) /

pré-op 44,19 kg/ $\mathrm{m} 2+110$ adultos

$\mathrm{kg} / \mathrm{m} 2+53$

pós $45,13 \mathrm{~kg} / \mathrm{m} 2$

174 adultos $(42,9$

Body Shape

Questionnaire

Pré-op / Pós-op1 mês - 24 meses /

Pós-op 24 meses em diante

Transtornos alimentares e Depressão

\section{Body Shape}

Questionnaire

Pré-operatório

Depressão

Sem diferenças significativas em relação à imagem corporal entre os grupos (1) abuso sexual e (2) sem relato de abuso no período de 6-18 meses, $t(27)=4,67$, $p=0,01$.

Diferenças significativas entre pacientes com perda de controle sobre a comida e sem perda de controle, com os primeiros apresentando escores mais elevados de prejuízo na imagem corporal e de sintomas de transtornos alimentares, $F(1,330)=7,79, p<0,01$, tanto no pré-operatório quanto no pós-operatório a longo prazo.

NA (estudo de estrutura fatorial e validade de construto).

Correlação positiva entre alta perda de peso corporal no pósop e menor rejeição corporal do próprio corpo e com melhor dinâmica corporal vital $(p=0,0001)$. 


\begin{tabular}{|c|c|c|c|}
\hline Estudo & Amostra & Instrumentos de IC & $\begin{array}{c}\text { Momento } \\
\text { Avaliação IC }\end{array}$ \\
\hline $\begin{array}{l}\text { Kitzinger et } \\
\text { al., } 2012\end{array}$ & $\begin{array}{c}252 \text { adultos }(46,1 \\
\pm 12,4 \text { anos }) / \\
\text { IMC médio no } \\
\text { pré-operatório } \\
47,5 \mathrm{~kg} / \mathrm{m} 2, \text { pós- } \\
\text { operatório } 30,5 \\
\mathrm{~kg} / \mathrm{m} 2\end{array}$ & $\begin{array}{l}\text { Post-Bariatric } \\
\text { Satisfaction } \\
\text { Questionnaire }\end{array}$ & $\begin{array}{c}\text { Pós-cirurgia pelo } \\
\text { menos } 1 \text { ano }\end{array}$ \\
\hline $\begin{array}{l}\text { Koller et al., } \\
2013\end{array}$ & $\begin{array}{c}27 \text { adultos }(39,9 \\
\text { anos) / IMC } \\
\text { médio pré- } \\
\text { operatório } 46,7 \\
\mathrm{Kg} / \mathrm{m} 2 . \text { Peso } \\
\text { médio no pós- } \\
\text { operatório } 71 \mathrm{~kg}\end{array}$ & $\begin{array}{l}\text { Body Appraisal } \\
\text { Inventory }\end{array}$ & $\begin{array}{l}\text { Pré-op } 2 \text { semanas } \\
\text { / Pós-op } 6 \text { meses }\end{array}$ \\
\hline $\begin{array}{l}\text { Munoz et al., } \\
2010\end{array}$ & $\begin{array}{l}57 \text { adultos }(39 \pm \\
4,3 \text { anos) / IMC } \\
\text { médio no pré-op } \\
52,54 \mathrm{~kg} / \mathrm{m} 2\end{array}$ & $\begin{array}{c}\text { Silhouette Figure } \\
\text { Rating Scale }\end{array}$ & $\begin{array}{l}\text { Pré-op / Pós- } \\
\text { operatório } 1 \text { ano }\end{array}$ \\
\hline $\begin{array}{l}\text { Ortega et al., } \\
2012\end{array}$ & $\begin{array}{c}60 \text { adultos / IMC } \\
\text { no pré-op 44,9 } \\
\pm 6,3 \mathrm{~kg} / \mathrm{m} 2 / \mathrm{IMC} \\
\text { médio no pós-op } \\
6 \text { meses } 34,1 \pm \\
4,7 \mathrm{~kg} / \mathrm{m} 2 / \mathrm{IMC} \\
\text { médio no pós } 1 \\
\text { ano } 29,9 \pm 4,8 \\
\mathrm{~kg} / \mathrm{m} 2\end{array}$ & $\begin{array}{c}\text { Body Shape } \\
\text { Questionnaire }\end{array}$ & $\begin{array}{l}\text { Pré-op / Pós-op } \\
6 \text { meses / Pós- } \\
\text { operatório } 1 \text { ano }\end{array}$ \\
\hline
\end{tabular}

Depressão, Ansiedade e Transtorno ObsessivoCompulsivo.

Ribeiro et al., 2013

423 adultos

Sarwer et al., 250 adultos / IMC 2013 médio 44,6 Kg/ $\mathrm{m} 2$.

Body Shape Questionnaire
Pré-operatório / Pós-operatório cinco grupos: 1) pré-operatório, 2)

Escala de Desenhos de Silhuetas pós-operatório 12 meses, 3) pósoperatório 12-24 meses, 4) pósoperatório 25-36 meses, 5) + de 37 meses.
NA

Disfunção sexual e Depressão
Após perda de peso provocada pela cirurgia, ambos os sexos estão insatisfeitos com as áreas do corpo, como abdômen / cintura, peito e coxas.

Perda de peso após a cirurgia melhora a qualidade de vida e imagem corporal $(p<$ $0,05)$

Diminuição significativa na diferença entre a forma corporal ideal e a forma corporal atual $(t$ $=2,63, p=0,02)$ após a cirurgia.

Imagem corporal negativa se correlacionou com transtorno obsessivo-compulsivo, depressão, ansiedade, índice geral de angústia $(p<0,001)$, ideação paranóica $(p<$ $0,05)$ e psicoticismo ( $p$ $<0,01)$ um ano após cirurgia.

Diferenças significativas no pré-op em relação a todos os grupos quanto à escolha de figuras maiores que representavam o próprio tamanho $(p<0,001)$. O grupo Pre-O também se diferenciou dos grupos PO-1, PO-2 e PO-3, mostrando tendência à escolha de silhuetas maiores depois da operação $(p<0,001)$. Em relação ao tamanho que gostariam de ter, o grupo PO-4 se diferenciou de PO-1 e PO-2 $(p<0,05)$, com tendência à escolha de figuras maiores.

Não houve diferenças significativas. 


Estudo Amostra $\quad$ Instrumentos de IC $\begin{gathered}\text { Momento } \\ \text { Avaliação IC }\end{gathered}$ Comorbidades $\quad$ Resultados IC

Sousa et al., 52 adultos $(44,04$

2014 anos), NI*
Pós-operatório (mais de 2 anos)

Pré-op / Pós-op

(2 anos após a cirurgia) $\mathrm{kg} / \mathrm{m} 2$

129 adultos $(39,6$ Wimmelmann $\pm 9,5$ anos)/ IMC et al., 2014 no pré $42,91 \pm$ $5,77 \mathrm{~kg} / \mathrm{m} 2$

$\begin{array}{lc} & 393 \text { adultos } \\ \text { Zwaan et al., } & (41,63 \text { anos }) / \\ 2014 & \text { IMC no pré-op } \\ & 48,74 \mathrm{~kg} / \mathrm{m} 2\end{array}$

Body Image Questionnaire
Pré-op 12 meses aproximadamente
Transtornos

Alimentares,

Ansiedade

social e

Neuroses

Personalidade

e Diabetes tipo 2
A ingestão alimentar compulsiva, a \% de IMC ganho após cirurgia e a preocupação com a imagem corporal explicam $50 \%$ da variância da sintomatologia depressiva após a cirurgia $(\mathrm{R} 2=0,46)$, sendo significativo $F$ $(3,43)=14,117, \quad p<$ 0,001 . Maior insatisfação com a imagem corporal estava associada a mais sintomas depressivos, $\beta=0,535, t=3,71, p=$ 0,001 .

A imagem corporal explicou mais do que $25 \%$ da variância em dois anos pós-op da mudança na atitude corporal $(p<$ 0,001 )

Pacientes com diabetes tipo 2 apresentaram melhor imagem corporal do que os pacientes sem diabetes $(p<0,05)$

Pacientes após cirurgia de contorno corporal relataram melhor avaliação da aparência, satisfação da área do corpo e funcionamento físico $(p<0,001)$

Nota. NI*: Não informado. NA: Não avaliado.

Outros instrumentos foram utilizados nos artigos selecionados. O Body Image Questionnaire (Clement \& Loewe, 1996), que investiga a aparência externa e o sentimento de bem estar com o próprio corpo e os componentes enérgicos e de movimento da imagem corporal. A Body Image State Scale (Cash \& Pruzinsky, 2002), que avalia as experiências afetivas momentâneas em relação à aparência física do indivíduo. A Body Image Inventory (Koller et al., 2013), que avalia aspectos subjetivos negativos em relação ao corpo, além de avaliar a percepção de dinâmica e a vitalidade corporal. A Body Appraisal Inventory
(Strauß \& Appelt, 1983), que busca examinar insegurança, desconforto, atratividade, auto-confiança, acentuação e sensibilidade em relação ao próprio corpo dos participantes. O Body Attitude Test (Probst, Vandereycken, van Coppenolle, \& Vanderlinden, 1995), que foi desenvolvido para medir a experiência subjetiva e a atitude para com o próprio corpo. Por fim, a Multidimensional Body-Self Relations Questionnaire (Cash, 1991), que investiga dimensões de relação com a própria imagem corporal, como a importância da aparência percebida e sua influência no comportamento do indivíduo. 
O estudo de Kitzinger et al. (2012) desenvolveu uma escala para o próprio estudo, a Post-Bariatric Satisfaction Questionnaire, com foco principal nas preocupações estéticas e contorno corporal após a cirurgia de redução de peso. Dos nove instrumentos para avaliação de imagem corporal identificados na revisão, o único que avalia a dimensão perceptiva da imagem corporal é a Escala de Figura de Silhuetas. Os outros oito instrumentos medem a atitude e o nível de satisfação com o próprio corpo.

Os artigos selecionados mostraram diferentes estratégias em relação ao momento de avaliação da imagem corporal. Em cerca de metade dos estudos a avaliação ocorreu em duas etapas, antes e depois da cirurgia $(n=8)$. Os outros artigos se dividiram entre avaliações apenas pré-cirúrgicas $(n=3)$ ou somente pós-cirúrgicas $(n=4)$. Não se observou padronização sobre o momento preciso em que se avaliou a imagem corporal na pré-cirurgia. Os artigos que especificaram tal informação variaram entre duas semanas a 12 meses antes da cirurgia, indicando amplitude de tempo de avaliação e baixa homogeneidade sobre critérios de mensuração. Dentre os artigos que realizaram avaliação no pós-cirúrgico, todos definiram o momento da avaliação, porém com elevada variação temporal, entre um mês a 12 anos, assim como observado na avaliação pré-cirúrgica.

Dentre os 15 artigos selecionados, 10 investigaram comorbidades associadas à obesidade. A maioria $(n=8)$ avaliou sintomas de depressão, seguida por transtornos alimentares $(n=3)$ e ansiedade $(n=3)$. Apenas dois dos 10 estudos que investigaram comorbidades encontraram associações entre transtornos psiquiátricos e imagem corporal. Sousa et al. (2014) evidenciaram que a preocupação com a imagem do corpo somada à ingestão alimentar compulsiva e à porcentagem de IMC adquirida após cirurgia explicam 50\% da variância da sintomatologia depressiva no pós-cirúrgico. Já o estudo de Ortega et al. (2012), identificou que imagem corporal negativa no pré-cirúrgico correlaciona-se com sintomas psiquiátricos de transtorno obsessivo-compulsivo, depressão, ansiedade, índice geral de angústia, ideação paranóide e psicoticismo um ano após a cirurgia.

Todos os estudos que mensuraram avaliação da imagem corporal antes e depois da cirurgia $(n=9)$ apresentaram diferenças significativas da variável no pós-operatório. De forma geral, as diferenças estão relacionadas no pós-cirúrgico à maior satisfação com a imagem do próprio corpo, à diminuição da diferença de percepção entre a forma corporal atual e ideal, à escolha de silhuetas menores representando o próprio tamanho e à melhor avaliação da aparência, satisfação com áreas do corpo e o funcionamento físico.

\section{Discussão}

A revisão identificou que, embora ocorra avaliação da imagem corporal em indivíduos candidatos à cirurgia de redução de peso, há prevalência de foco na avaliação da dimensão atitudinal do constructo. A dimensão atitudinal foi observada na descrição dos artigos como mensuração da satisfação em relação ao próprio corpo. Avaliações da dimensão perceptiva da imagem corporal, caracterizada nos estudos como percepção do tamanho de silhuetas ou figuras corporais, foram menos frequentes. Tavares, Campana e Campana (2010) corroboram o achado de que são poucos os estudos que investigam efetivamente a dimensão perceptiva da imagem corporal na literatura. Por outro lado, em pesquisas com pacientes diagnosticados com Bulimia Nervosa e Anorexia Nervosa o uso de medidas perceptivas de imagem corporal parece ser mais frequente (Saikali, Soubhia, Scalfaro, \& Cordás, 2004).

Essa diferença de uso remete provavelmente ao valor diferencial do diagnóstico pela mensuração de aspectos perceptivos entre populações de obesos e indivíduos com transtornos alimentares. Não está claro na literatura sobre obesidade se funções perceptivas são comprometidas pelo acúmulo de peso. Contudo, uma mudança rápida de tamanho corporal, como observado nos resultados das cirurgias de redução de peso, poderia acarretar em mudanças importantes perceptivas 
e proprioceptivas. Tal hipótese justificaria o aumento da investigação pré e pós-cirúrgica tanto da dimensão perceptiva quanto atitudinal da imagem corporal. $\mathrm{O}$ valor da variável em um protocolo de avaliação pode ser exemplificado pelo estudo de Simonsen, Hundrup, Obel, Gronbaek e Heitmann (2008). Os pesquisadores mostraram que indivíduos com IMC elevado e com imagem corporal distorcida terão mais prejuízos para sua saúde do que aqueles com apenas IMC elevado. Ou seja, indivíduos obesos com o mesmo peso terão desfechos de saúde diferentes a depender da maneira como percebem o próprio corpo.

Sobre esse aspecto, destacou-se na revisão a heterogeneidade nos momentos de avaliação da imagem corporal no contexto de cirurgia de redução de peso. Embora mais da metade dos estudos tenha realizado a avaliação antes e depois da cirurgia, o momento específico dessa avaliação não foi homogêneo, variando tanto no pré quanto no pós-operatório. Na construção de protocolos de avaliação psicológica, as janelas de mensuração de variáveis são um fator crucial para a comparabilidade entre estudos e desenvolvimento de protocolos mais objetivos e precisos (American Educational Research Association [AERA], APA, \& National Council on Measurement in Education [NCME], 2014). Tal comparabilidade auxilia na garantia de validade das avaliações, fator de extrema relevância durante o desenvolvimento e a aplicação de testes psicológicos, principalmente quando utilizados para fins diagnósticos. Uma hipótese que pode explicar esta variabilidade nos momentos de avaliação da imagem corporal, conforme constatado na revisão, é a tendência mais exploratória dos artigos selecionados sobre a associação entre imagem corporal e desfechos clínicos.

Ainda que exploratórias, as intervenções de redução de peso mostraram-se correlacionadas às mudanças na imagem corporal dos indivíduos. Os estudos em geral revelaram melhorias tanto na dimensão atitudinal quanto na perceptiva após o procedimento cirúrgico. Apenas um artigo identificou insatisfação com algumas áreas do corpo no pós-operatório (Kitzinger et al.,
2012). Além disso, a imagem corporal negativa foi apontada como uma variável significativa associada à depressão, ansiedade e transtornos alimentares. Percebe-se dessa forma que a imagem corporal possui um papel relevante no prognóstico de intervenções cirúrgicas (Koller et al., 2013), muito embora na revisão um terço dos artigos não avaliou comorbidades da obesidade. Esperava-se um maior número de artigos porque a imagem corporal é uma variável de pesquisa prioritariamente psicológica (Saur, 2007) e os estudos selecionados para análise possuíam um caráter de avaliação de saúde mental.

A presente revisão encontrou resultados semelhantes ao estudo de Pull e Aguayo (2011) quanto à heterogeneidade de definições para a operacionalização da imagem corporal na pesquisa. Isto se reflete principalmente no elevado número de instrumentos que se dedicam à avaliação de imagem corporal. Tal achado mostra que não houve muito avanço na literatura em relação à conceptualização e mensuração da imagem corporal nos últimos quatro anos. Nesse sentido, a tarefa de comparação de resultados das pesquisas que avaliam imagem corporal permanece difícil.

Apesar do destaque concedido pela literatura ao impacto que a alteração da imagem corporal provoca em obesos mórbidos, a atual revisão revelou que a avaliação da variável não é uniforme. Todavia há uma tendência de avaliação do nível de satisfação subjetiva com o próprio corpo, mesmo que com diferentes instrumentos. Poucos estudos avaliaram os aspectos psicofísicos da percepção de imagem corporal. Uma das limitações da revisão foi avaliar apenas artigos publicados nas línguas portuguesa e inglesa, não abrangendo pesquisas sobre o tema em outros idiomas. A baixa homogeneidade de definições operacionais para imagem corporal pode estar na base de outra limitação da revisão, que foi definir os critérios de busca de artigos apenas com o termo imagem corporal. Futuras análises devem explorar a diferença entre protocolos de avaliação de imagem corporal entre obesos e outras populações clínicas em que esta variável possui papel prognóstico mais bem delimitado. 


\section{Referências}

Almeida, S. S., Zanatta, D. P., \& Rezende, F. F. (2012). Imagem corporal, ansiedade e depressão em pacientes obesos submetidos à cirurgia bariátrica. Estudos de Psicologia (Natal), 17(1), 153 160. doi:10.1590/S1413-294X2012000100019

Altabe, M. N., \& Thompson, J. K. (1996). Body image: A cognitive self-schema construct? $\operatorname{Cog}$ nitive Therapy and Research, 20(2), 171-193. doi:10.1007/BF02228033

American Educational Research Association, American Psychological Association, \& National Council on Measurement in Education. (2014). Standards for Educational and Psychological Testing. Washington, DC: American Educational Research Association Publications Sales.

Bruce, D. M., \& Mitchell, A. I. (2014). Surgery for obesity. Medicine, 43(2), 101-103. doi:10.1016/j. mpmed.2014.11.001

Buchwald, H., Avidor, Y., Braunwald, E., Jensen, M. D., Pories, W., Fahrbach, K., \& Schoelles, K. (2004). Bariatric surgery: A systematic review and meta-analysis. The Journal of the American Medical Association, 292(14), 1724-1737. doi:10.15406/aowmc.2015.02.00011

Buser, A. T., Lam, C. S., \& Poplawski, C. S. (2009). A long-term cross-sectional study on gastric bypass surgery: Impact of self-reported past sexual abuse. Obesity Surgery, 19(4). doi:10.1007/s11695-008-9555-2

Carr, D., \& Jaffe, K. (2012). The psychosocial consequences of weight change trajectories: Evidence from quantitative and qualitative data. Economics and Human Biology, 10(4), 419-430. doi:10.1016/j.ehb.2012.04.007

Cash, T. F. (1991). Binge-eating and body images among the obese: A further evaluation. Journal of Social Behavior and Personality, 6, 367-376.

Cash, T. F., \& Pruzinsky, T. (2002). Body image: A handbook of theory, research, and clinical practice. New York: Guilford Press. doi:10.1056/ NEJM200304033481428

Clement, U., \& Löwe, B. (1996). Validation of the FKB-20 as scale for the detection of body image distortions in psychosomatic patients. Psychotherapie, Psychosomatik, Medizinische Psychologie, 46(7), 254-259.
Conceição, E., Bastos, A. P., Brandão, I., Vaz, A. R., Ramalho, S., Arrojado, F., ...Machado, P. P. (2013). Loss of control eating and weight outcomes after bariatric surgery: A study with a Portuguese sample. Eating and Weight Disorders, 19(1), 103-109. doi:10.1007/s40519-0130069-0

Conde, W. L., \& Borges, C. (2011). O risco de incidência e persistência da obesidade entre adultos Brasileiros segundo seu estado nutricional ao final da adolescência. Revista Brasileira de Epidemiologia, 14(1), 71-79. doi:10.1590/S1415$-790 \times 2011000500008$

Cooper, P. J., Taylor, M. J., Cooper, Z., \& Fairburn, C. J. (1987). The development and validation of the Body-Shape Questionnaire. International Journal of Eating Disorders, 6(4), 485-494.

Costa, A. B., \& Zoltowski, A. P. C. (2014). Como escrever um artigo de revisão sistemática. In $\mathrm{S}$. H. Koller, M. C. P. de Paula Couto, \& J. V. Hohendorff (Eds.), Manual de Produção Científica (pp. 55-70). Porto Alegre, RS: Penso.

Dhurandhar, E. J., \& Keith, S. W. (2014). The aetiology of obesity beyond eating more and exercising less. Best Practice \& Research Clinical Gastroenterology, 28(4), 533-544. doi:10.1016/j. bpg.2014.07.001

Fabricatore, A. N., Crerand, C. E., Wadden, T. A., Sarwer, D. B., \& Krasucki, J. L. (2006). How do mental health professionals evaluate candidates for bariatric surgery? Survey results. Obesity Surgery, 16(5), 567-573.

Finer, N. (2011). Medical consequences of obesity. Medicine Journal, 39(1), 18-23. doi:10.1016/j. mpmed.2010.11.008

Friedman, M. A., \& Brownell, K. D. (1995) Psychological correlates of obesity: Moving to the next research generation. Psychological Bulletin, 117(1), 3-20. doi:10.1037//0033-2909.117.1.3

Gianos, M., Abdemur, A., Fendrich, I., Gari, V., Szomstein, S., \& Rosenthal, R. J. (2012). Outcomes of bariatric surgery in patients with body mass index $<35 \mathrm{~kg} / \mathrm{m} 2$. Surgery for Obesity and Related Diseases, 8(1), 25-30. doi:10.1016/j. soard.2011.08.012

Grilo, C. M., Henderson, K. E., Bell, R. L., \& Crosby, R. D. (2013). Eating disorder examination-questionnaire factor structure and construct validity in bariatric surgery candidates. Obesity Surgery, 23(5). doi:10.1007/s11695-012-0840-8 
Guh, D. P., Zhang, W., Bansback, N., Amarsi, Z., Birmingham, C. L., \& Anis, A. H. (2009). The incidence of co-morbidities related to obesity and overweight: A systematic review and meta-analysis. BMC Public Health, 88(9), 1-20. doi:10.1186/1471-2458-9-88

Holsen, I., Carlson, D. J., \& Skogbrott, M. B. (2012). Body image satisfaction among Norwegian adolescents and young adults: A longitudinal study of the influence of interpersonal relationships and BMI. Body Image, 9(2). doi:10.1056/ NEJM200304033481428

Hu, F. B. (2008). Obesity epidemiology. New York: Oxford University Press

Kakeshita, I. S., Silva, A. I. P., Zanatta, D. P., \& Almeida, S. S. (2009). Construção e fidedignidade teste-reteste de escalas de silhuetas brasileiras para adultos e crianças. Psicologia: Teoria $e$ Pesquisa, 25, 263-270.

Kinzl, J. F., Lanthaler, M., Stuerz, K., \& Aigner, F. (2011). Long-term outcome after laparoscopic adjustable gastric banding for morbid obesity. Eating Weight Disorders, 16(4), 250-256. doi: $10.3275 / 7752$

Kinzl, J. F., Schrattenecker, M., Traweger, C., Mattesich, M., Fiala, M., \& Biebl, W. (2006). Psychosocial predictors of weight loss after bariatric surgery. Obesity Surgery, 16(12), 1609-1614. doi:10.1381/096089206779319301

Kitzinger, H. B., Abayev, S., Pittermann, A., Karle, B., Kubiena, H., Bohdjalian, A., ...Frey, M. (2012). The prevalence of body contouring surgery after gastric bypass surgery. Obesity Surgery, 22(1), 8-12. doi:10.1007/s11695-0110459-1

Koller, M., Schubhart, S., \& Hintringer, T. (2013). Quality of life and body image after circumferential body lifting of the lower trunk: A prospective clinical trial. Obesity Surgery, 23(4), 561-566. doi:10.1007/s11695-012-0849-z

Liechty, J. M., \& Lee, M. J. (2015). Body size estimation and other psychosocial risk factors for obesity onset among US adolescents: Findings from a longitudinal population level study. International Journal of Obesity, 39. doi:10.1038/ijo.2014.191

Maggard, M. A., Shugarman, L. R., Suttorp, M., Maglione, M., Sugerman, H. J., Livingston, E. H., ...Shekelle, P. G. (2005). Meta-analysis: Surgical treatment of obesity. Annals of Internal
Medicine, 142(7), 547-584. doi:10.7326/00034819-142-7-200504050-00013

Munoz, D., Chen, E. Y., Fischer, S., SanchezJohnsen, L., Roherig, M., Dymek-Valentine, M., ...Le Grange, D. (2010). Changes in desired body shape after bariatric surgery. Eating Disorders, 18(4), 347-354. doi:10.1080/106402 66.2010 .490126

Ortega, J., Fernandez-Canet, R., Alvarez-Valdeita, S., Cassinello, N., \& Baguena-Puigcerver, M. J. (2012). Predictors of psychological symptoms in morbidly obese patients after gastric bypass surgery. Surgery for Obesity and Related Diseases, 8(6), 770-776. doi:10.1016/j.soard.2011.03.015

Picot, J., Jones, J., Colquitt, J. L., Gospodarevskaya, E., Loveman, E., Baxter, L., \& Clegg, A. J. (2009). The clinical effectiveness and costeffectiveness of bariatric (weight loss) surgery for obesity: A systematic review and economic evaluation. Health Technology Assessment, 13(41), doi:10.3310/hta13410

Popkin, B. M. (2007). Global context of obesity. In S. Kumanyika \& R. Brownson, Handbook of Obesity Prevention: A resource for health professionals (pp. 227-238). New York: Springer.

Probst, M., Vandereycken, W., van Coppenolle, H., \& Vanderlinden, J. (1995). The body attitude test for patients with an eating disorder: Psychometric characteristics of a new questionnaire. Eating Disorders, 3(2), 133-144.

Pull, C. B. (2010). Current psychological assessment practices in obesity surgery programs: What to assess and why. Current Opinion in Psychiatry, 23(1), 30-36. doi:10.1097/ YCO.0b013e328334c817

Pull, C. B., \& Aguayo, G. A. (2011). Assessment of body-image perception and attitudes in obesity. Current Opinion in Psychiatry, 24(1). doi:10.1097/YCO.0b013e328341418c

Ribeiro, G. A. N. A., Giampietro, H. B., Barbieri, L. B., Pacheco, R. G., Queiroz, R., \& Ceneviva, R. (2013). Percepção corporal e cirurgia bariátrica: $\mathrm{O}$ ideal e o possível. Arquivos Brasileiros de Cirurgia Digestiva 26(2), 124-128.

Saikali, C. J., Soubhia, C. L., Scalfaro, B. M., \& Cordás, T. A. (2004). Imagem corporal nos transtornos alimentares. Revista de Psiquiatria Clínica, 31(4), 164-166.

Salihu, H. M., Bonnema, S. M., \& Alio, A. P. (2009). Obesity: What is an elderly population growing 
into? Maturitas, 63(1), 7-12. doi:10.1016/j.maturitas.2009.02.010

Sarwer, D. B., Spitzer, J. C., Wadden, T. A., Rosen, R. C., Mitchell, J. E., Lancaster, K., ...Christian, N. J. (2013). Sexual functioning and sex hormones in persons with extreme obesity and seeking surgical and nonsurgical weight loss. Surgery for Obesity and Related Diseases: Official Journal of the American Society for Bariatric Surgery, 9(6), 997-1007. doi:10.1016/j. soard.2013.07.003

Saur, A. M. (2007). Imagem corporal: Auto-satisfação e representação psíquica em Desenhos da Figura Humana (Dissertação de mestrado, Universidade de São Paulo, Ribeirão Preto, SP, Brasil)

Simonsen, M. K., Hundrup, Y. A., Obel, E. B., Gronbaek, M., \& Heitmann, B. L. (2008). Intentional weight loss and mortality among initially healthy men and women. Nutrition Reviews, 66(7), 375386. doi:10.1111/j.1753-4887.2008.00047.x

Skelton, J. A., Cook, S. R., Auinger, P., Klein, J. D., $\&$ Barlow, S. E. (2009). Prevalence and trends of severe obesity among US children and adolescents. Academic Pediatrics, 9(5), 322-329. doi:10.1016/j.acap.2009.04.005

Skelton, J. A., Irby, M. B., Grywacz, J. G., \& Miller, G. (2011). Etiologies of obesity in children: Nature and nurture. Pediatric Clinics of North America, 58(6), 1333-1354. doi:10.1016/j. pcl.2011.09.006

Sousa, P., Bastos, A. P., Venancio, C., Vaz, A. R., Brandão, I., Costa, J. M., ...Conceição, E. (2014). Compreender a sintomatologia depressiva após a cirurgia bariátrica: $\mathrm{O}$ papel do peso, da alimentação e da imagem corporal. Acta Médica Portuguesa, 27(4), 450-457.

Strauß, B., \& Appelt, H. (1983). Ein Fragebogen zur Beurteilung des eigenen Körpers. Diagnostica, 29, 145-164.

Stunkard, A. J., Sorenson, T., \& Schlusinger, F. (1983). Use of the Danish adoption register for the study of obesity and thinness. In S. S. Kety, L. P. Rowland, R. L. Sidman, \& S. W. Mathysse (Eds.), The genetics of neurologic and psychiatric disorders (pp. 115-120). New York: Raven.
Tavares, M. C. G. C. F., Campana, A. N. N. B., Filho, R. F. T., \& Campana, M. B. (2010). Avaliação perceptiva da imagem corporal: História, reconceituação e perspectivas para o Brasil. Psicologia em Estudo, 15(3), 509-518.

Thompson, J. K. (2004). The (mis)measurement of body image: Ten strategies to improve assessment for applied and research purposes. Body Image, 1(1), 7-14. doi:10.1016/S17401445(03)00004-4

Van Hout, G. C., Hagendoren, C. A.,Verschure, S. K., \& van Heck, G. L. (2009). Psychosocial predictors of success after vertical banded gastroplasty. Obesity Surgery, 19, 701-707. doi:10.1007/ s11695-008-9446-6

Wimmelmann, C. L., Dela, F., \& Mortensen, E. L. (2014). Psychological predictors of weight loss after bariatric surgery: A review of the recent research. Obesity Research \& Clinical Practice, 8(4), 299-313. doi:10.1016/j.orcp.2013.09.003

World Health Organization. (1997). Obesity. Preventing and managing the global epidemic (Report of a WHO Consultation on Obesity). Geneva, Switzerland: Author.

Zwaan, M., Georgiadou, E., Stroh, C. E., Teufel, M., Köhler, H., Tengler, M., \& Müller, A. (2014). Body image and quality of life in patients with and without body contouring surgery following bariatric surgery: A comparison of pre- and post-surgery groups. Frontiers in Psychology, 18(5), 1310. doi:10.3389/fpsyg.2014.01310
Recebido: 09/10/2015

$1^{a}$ revisão: $14 / 01 / 2016$ Aceite final: 14/01/2016 